\title{
Amisulpride as adjunct to clozapine in treatment-resistant schizophrenia and schizoaffective disorder An open-label pilot study
}

Liezl Koen, MMed (Psych)

Dana J Niehaus, DMed

Angelica Schulte, MMed (Psych)

Jaqueline Muller, MMed (Psych)

Robin Emsley, DMed

Piet Oosthuizen, DMed

Department of Psychiatry, Stellenbosch University, W Cape

Background. Although clozapine is the treatment of choice for treatment-resistant schizophrenia, many patients remain symptomatic despite adequate treatment with this medication. One suggested strategy to improve efficacy has been the addition of a potent $D_{2}$ blocker such as amisulpride to treatment with clozapine.

Methods. In this study, 20 subjects who were treatment-resistant to conventional antipsychotics and who were still symptomatic despite adequate treatment with clozapine received amisulpride as adjunctive treatment. After baseline assessment all subjects were titrated to amisulpride $400 \mathrm{mg}$ per day and then reassessed after 8 weeks by a blinded rater.

Results. Analysis showed statistically significant improvements in PANSS (Positive and Negative Symptom Scale) total score ( $t=$ 3.49, $d f=18, p=0.003)$, PANSS negative subscale score $(t$ $=3.22, \mathrm{df}=18, p=0.0051$, and PANSS depression factor score $(t=3.89, d f=19, p=0.001)$

Discussion. This study suggests that addition of the secondgeneration antipsychotic amisulpride to a stable treatment regimen with clozapine may offer additional benefits in terms of negative and depressive symptoms.

Despite its reported prevalence of only $1 \%$ in the general population, more than 10\% of disabled individuals in the USA have schizophrenia, making it one of the leading causes of disability and health care expenditure in the world.' The introduction of conventional antipsychotics in the 1950s represented a remarkable turning point in the treatment of psychotic illness. Approximately $70 \%$ of patients show substantial symptom reduction in the short term with pharmacological treatment. ${ }^{2}$ Unfortunately, this short-term efficacy has not translated into long-term success, as the majority of patients show only partial response and experience persistent positive and/or negative symptoms over time. ${ }^{3}$ Between 10\% and 20\% of patients are completely resistant to treatment with first-generation antipsychotics. ${ }^{4}$

Another revolution in the treatment of psychotic illness came with the introduction of a series of second-generation antipsychotics. Although some studies suggest that they may offer additional benefits in treatment-resistant patients, their major advantage seems to be in reduction of the burden of side-effects. ${ }^{5}$ Data on their effect in treatment-resistant patients remain inconclusive.

At this time clozapine, the prototypical 'atypical' antipsychotic, is the only medication with an established record of efficacy against refractory symptoms. ${ }^{4}$ However, many patients are also clozapineresistant and remain unresponsive despite treatment with high doses of this medication. ${ }^{6}$ Although a recent review found only 11 published randomised, double-blind, placebo-controlled augmentation trials in clozapine-resistant patients, ${ }^{7}$ many different augmentation strategies have been employed (including other antipsychotic medications, mood stabilisers, antidepressants, electroconvulsive therapy and others). While a number of these strategies have been shown to have some benefit, so far none have proved to be widely effective. ${ }^{8}$

One of the strategies for augmentation has been to increase dopamine $D_{2}$ receptor occupancy by addition of a $D_{2}$ blocker. Shiloh et al. ${ }^{9}$ reported a study of 28 patients where sulpiride was used as augmenting agent, with improvement in symptoms superior to placebo.

Amisulpride is a second-generation antipsychotic that binds selectively to dopaminergic $D_{2} / D_{3}$ receptor subtypes. It has no affinity for $D_{1}, D_{4}$ and $D_{5}$ receptors or for $\alpha$-adrenergic, histamine $\mathrm{H}_{1}$ and cholinergic receptors and only a small clinically insignificant affinity for serotonin receptors. Amisulpride has been reported to have a higher affinity for dopamine receptors in the limbic structures than dopamine receptors in the striatum. ${ }^{10}$ 
At low doses it is reported to selectively block presynaptic $D_{2} / D_{3}$ receptors, resulting in increased release of dopamine. Amisulpride has been shown to be as effective as first-generation antipsychotics ${ }^{11}$ and other second-generation antipsychotics ${ }^{12}$ in the treatment of acute exacerbations of schizophrenia. A significant effect on negative symptoms has also been reported, particularly at lower doses. ${ }^{13}$

The purpose of this study was to examine the efficacy of augmenting clozapine treatment with amisulpride in a cohort of subjects with clozapine-resistant schizophrenia. The rationale for using amisulpride as add-on lies in its unique receptor profile allowing it to be regarded as a particularly advantageous addon drug in combination with a multireceptor substance such as clozapine, as additive effects only appear in terms of dopamine receptors and not in terms of muscarinergic, adrenergic or histaminergic receptors where addition would just lead to an increased side-effect burden.

\section{Method}

Study participants were a random sample of 20 subjects with treatment-resistant schizophrenia $(N=16)$ or schizoaffective disorder $(N=4)$. Subjects were recruited from the inpatient wards of Stikland Hospital in Cape Town. The study protocol and patient information and consent procedures were approved by Stellenbosch University Ethics Committee, and all subjects provided written informed consent to participate.

The study included male and female patients between the ages of 18 and 65 years (extremes included), with a Diagnostic and Statistical Manual of Mental Disorders (DSM-M diagnosis of schizophrenia or schizoaffective disorder. All subjects had to be treatment-resistant on clozapine. Initial treatment resistance was defined as continued psychosis with ongoing functional impairment for a period of at least 1 year (in all but 2 cases this period had in fact been 5 years or longer) during which time the patient had been subjected to at least two adequate trials with antipsychotics (other than clozapine) from different classes, each over a trial period of at least 6 weeks. Treatment with both these medications had to be at a dosage equivalent to at least $10 \mathrm{mg}$ haloperidol per day or had to have been discontinued because of intolerable side-effects, after which clozapine was initiated. To be considered for the trial, the daily dosage of clozapine had to be at least $400 \mathrm{mg} /$ day, or the maximum tolerated dose, for at least 6 weeks. Furthermore, no change in dose of any medication was allowed for 6 weeks before inclusion. Clozapine treatment- resistant psychosis was defined as a score of at least 4 on at least 1 item of the positive subscale of the Positive and Negative Syndrome Scale (PANSS)..$^{14}$

Exclusion criteria were: (i) subjects not stabilised on their current treatment, i.e. subjects who were considered to be still improving/deteriorating on treatment with clozapine; and (ii) subjects with unstable neurological or physical disease.

All the patients had been inpatients for at least 10 weeks before inclusion in the study and there was no history of current substance abuse or dependence. All ratings were done by a single, blinded rater (AS). AS took no part in the treatment of the subjects before or during the trial and was not aware of the dose of amisulpride that subjects received. Baseline demographic data were obtained and all subjects were rated using the PANSS. Although no fixed dose of amisulpride was defined in the protocol, in the event all subjects were titrated to 400 mg amisulpride within 3 days, after which the dose remained constant for the rest of the treatment period. AS was unaware of the fact that all subjects were receiving the same dose of adjunctive amisulpiride. Subjects were re-evaluated at 8 weeks using the same scales.

\section{Statistical analysis}

All analyses were performed using Statistica 6 software (StatSoft Inc. 1994 - 2003). As subjects only had two evaluations (baseline and endpoint), we did not use the last observation carried forward, but did a completers-only analysis. As it turned out, only 1 subject did not complete the study. The paired ttest was used to determine the significance of changes in continuous variables over the study period. Spearman's rank-order correlation coefficients were calculated to determine possible correlations between changes in continuous variables. Significance tests were performed at an alpha level of 0.05 .

\section{Results}

Twenty subjects were included in the study. Eighteen subjects (90\%) were male. The mean age at inclusion I \pm standard deviation (SD)l was 37.7 ( \pm 8.4) years. One subject did not complete the study because of extrapyramidal side-effects. The mean dosage for clozapine at baseline was 660 ( \pm 128.1) $\mathrm{mg} /$ day. Concomitant medications were sodium valproate 19 patients, dosage range 1000 - 2400 mg/day, mean dose $1555 \mathrm{mg} /$ day), lithium carbonate (2 patients, $750 \mathrm{mg}$ and $1250 \mathrm{mg}$ respectively) and phenytoin (1 patient, $300 \mathrm{mg} /$ day). Haloperidol (2.5 mg- 7.5 mg/day, mean dosage $4.375 \mathrm{mg} /$ 
dayl was withdrawn in the case of 4 patients before baseline. PANSS total and subscale scores at baseline and endpoint as well as the results of the paired ttest for each of the variables are presented in Table I. To further explore the change in the general psychopathology subscale and the effect of mood symptoms, we also evaluated the change in the PANSS depression factor, as described by Kay in his original factor analysis of the PANSS, 15 as well as the change in the general psychopathology subscale where the items of the depression factor (G1, G2, G3 and G6) were removed. Results are listed in Table II.

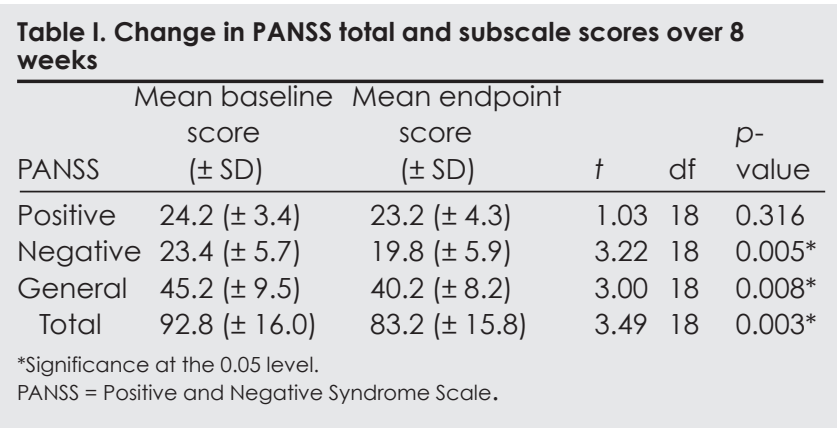

Table II. Change in PANSS depression factor score and general psychopathology score with depression factor items removed

\begin{tabular}{|c|c|c|c|c|c|}
\hline PANSS & $\begin{array}{l}\text { Mean } \\
\text { baseline } \\
\text { score } \\
( \pm S D)\end{array}$ & 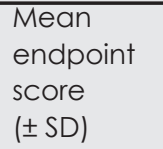 & $t$ & $d f$ & $\begin{array}{l}p- \\
\text { value }\end{array}$ \\
\hline $\begin{array}{l}\text { Depression } \\
\text { factor }\end{array}$ & & & & & \\
\hline $\begin{array}{l}\text { score } \\
\text { General } \\
\text { minus } \\
\text { depression } \\
\text { factor }\end{array}$ & $9.7( \pm 4.1)$ & $7.2( \pm 3.3)$ & 3.89 & 19 & $0.001^{*}$ \\
\hline score & $35.1( \pm 6.9)$ & $31.0( \pm 9.6)$ & 2.04 & 19 & 0.055 \\
\hline
\end{tabular}

Because the most significant changes were in the depression factor and the negative symptom subscale, we were interested to explore the possibilty that these were in fact reflecting the same changes, i.e. that the improvement in negative symptoms could merely be a reflection of the improvement in the depression factor, or vice versa. To determine this relationship we calculated Spearman's rank order correlation coefficients for the percentage change in negative symptoms over the 8 weeks and the percentage change in depression factor scores. The results showed that the correlation between these two variables was not statistically significant $(N=$ 19, Spearman's $r=0.40,+(N-2)=1.81, p=0.091$.

\section{Discussion}

The last decade has seen major developments in our understanding and treatment of schizophrenia. Not only has the introduction of the second-generation antipsychotics provided us with a hugely expanded armamentarium of treatment options, but research has also provided us with many new insights into the complexities of the disease and its clinical presentation. Long gone are the days when the focus of treatment was limited to the suppression of positive symptoms. Today we understand that there are many symptom complexes in the presentation of the disorder and that treatments that improve one set may not necessarily improve the others, and at times may even exacerbate them. Although treatment with a single agent remains the ideal, our current treatment options often fail to achieve an acceptable outcome.

It has been suggested before that amisulpride may have unique properties in the treatment of negative symptoms and mood symptoms of schizophrenia. ${ }^{10}$ Our study clearly supports this notion - whereas amisulpride did not offer any additional benefit in terms of treatment of positive symptoms in this treatment-resistant population who were already on high doses of clozapine over extended periods, all other subscales of the PANSS improved, with the greatest effect seen in negative symptoms and the depression factor of the PANSS. Although both these symptom domains improved, they were separate factors and did not influence each other significantly. Therefore the effect on the negative symptom scale cannot merely be interpreted as improvement in secondary negative symptoms as a result of improvement in mood symptoms.

The finding on the effect on the depression factor is similar to the results of a recent meta-analysis of the use of amisulpride in acute exacerbations of schizophrenia. ${ }^{16}$ Our study suggests that these effects are also achievable in patients regarded as treatment-resistant. There have been conflicting reports regarding the relationship between depressive symptoms and treatment outcome. However, it would appear that the presence of depressive symptoms in the acute phase of the illness might be associated with a favourable outcome. ${ }^{17}$ However, if these symptoms persist they appear appear to be negative prognostic indicators. ${ }^{18}$ It is clear, therefore, that treatment strategies in schizophrenia should always consider mood symptoms as an important focus of treatment.

The differentiation between primary and secondary negative symptoms continues to be a major challenge to researchers and 
clinicians alike. ${ }^{19}$ Although it would be premature to claim that the effect we saw was the result of an effect on 'core' negative symptoms, we can say with some degree of conviction that it was clearly not a result of improvement in mood symptoms. We did not formally record assessments of extrapyramidal side-effects, but it seems highly unlikely that the improvement we saw would have resulted from improvement in these side-effects. Further studies are needed to elucidate the effect of amisulpride on negative symptoms.

This study has a number of limitations. As it was a naturalistic pilot study, no control group was included and the number of subjects was small. However, there was only 1 dropout. Furthermore, a number of subjects were receiving concomitant medications, but

\section{References}

1. Rupp A, Keith SJ. The costs of schizophrenia. Psychiatr Clin North Am 1993; 16: 423 .

2. Wirshing WC, Marder SR, Van Putten T, Ames D. Acute treatment of schizophrenia. In: Bloom FE, Kupfer DJ, eds. Psychopharmacology: The Fourth Generation of Progress. New York: Raven Press, 1994.

3. Emsley RA. Partial response to antipsychotic treatment: The patient with enduring symptoms. J Clin Psychiatry 1999; 60: suppl.23, 10-13

4. Kane J, Honigfeld G Singer I. Meltzer HY and the Clozaril Collaborative Study Group. Clozapine for the treatment-resistant schizophrenic: a double-blind comparison with chlorpromazine. Arch Gen Psychiatry 1988; 45: 789-796.

5. Emsley R. Outcome of firstepisode schizophrenia and the new antipsychotics: $A$ literature review. S Afr Med / 1996: 86: 729-734.

6. Breier A, Buchanan RW, Kirkpatrick B, et al. Effect of clozapine on positive and negative symptoms in outpatients with schizophrenia. Am J Psychiatry 1994; 151: 20-26.

7. Kontaxakis VP, Ferentinos PP, Havaki-Kontaxaki BJ, Roukas DK. Randomized controlled augmentation trials in clozapine-resistant schizophrenic patients: a critical review. European Psychiatry 2005; 20: 409-415.

8. Conley RR, Buchanan RW. Evaluation of treatment resistant schizophrenia. Schizophr Bull 1997; 23: $663-674$.

9. Shiloh R, Zemishlany Z, Aizenberg D, et al. Sulpiride augmentation in people with schizophrenia partially responsive to clozapine. A double-blind, placebo-controlled study. BrJ Psychiatry 1997; 171: 569-573. it is important to note that with regard to ongoing medication no changes in dosage were made for at least 8 weeks before inclusion or at any time during the study. The treatment period was also fairly short, but this compared well with the treatment period in the study by Shiloh et al. ${ }^{9}$

Although the study results are fairly modest, there was a clear statistically significant improvement from baseline. Furthermore, it must be remembered that our study population was severely treatment-resistant. Also, the relatively low dose of amisulpride may have limited its effect on positive symptoms. However, this study does suggest that a novel antipsychotic, amisulpride, deserves attention as add-on medication in treatment-resistant populations.

10. Moller HJ. Amisulipride: a review of its efficacy in schizophrenia. Acta Psychiat Scand Supp/ 2000; 101: 17-22.

11. Moller HJ, Boyer P, Fleurot O, Rein W, and the PROD-ASLP study group. Improvement of acute exacerbations of schizophrenia with amisulpiride: a comparison with haloperidol. Psychopharmacology 1997; 132: 396-401.

12. Peuskens J, Bech P, Moller HJ. Amisulpiride vs. risperidone in the treatment of acute exacerbations of schizophrenia. Psychiatry Res 1999; 88: 107-117.

13. Pailliere-Martinot ML, Lecrubier Y, Martinot JL, Aubin F. Improvement of some schizophrenic deficit symptoms with low doses of amisulipride. Am J Psychiatry 1995; 152: 130-133.

14. Kay SR, Fizbein A, Opler LA. The Positive and Negative Syndrome Scale (PANSS) for schizophrenia. Schizophr Bull 1987; 13: 261-267.

15. Kay SR. Positive and Negative Syndromes in Schizophrenia: Assessment and Research. New York: Brunner/Mazel, 1991.

16. Peuskens 」, Moller HJ, Puech A. Amisulpiride improves depressive symptoms in acute exacerbations of schizophrenia: comparison with haloperidol and risperidone. Eur Neuropsychopharmacol 2002; 12: 305-310

17. Kay SR, Lindenmeyer J. Outcome predictors in acute schizophrenia: Prospective significance of background and clinical dimensions. J Nerv Ment Dis 1987; 175: 152-160.

18. Falloon I, Watt DC, Shepherd M. A comparative controlled trial of pimozide and fluphenazine decanoate in the continuation therapy of schizophrenia. Psychol Med 1978; 8: 59-70.

19. Kelley ME, van Kammen DP, Allen DN. Empirical validation of primary negative symptoms: independence from effects of medication and psychosis. Am J Psychiatry 1999; 156: 406-411. 